TITLE:

\title{
Thermal and shock induced modification inside a silica glass by focused femtosecond laser pulse
}

\section{AUTHOR(S):}

Sakakura, Masaaki; Terazima, Masahide; Shimotsuma, Yasuhiko; Miura, Kiyotaka; Hirao, Kazuyuki

\section{CITATION:}

Sakakura, Masaaki ... [et al]. Thermal and shock induced modification inside a silica glass by focused femtosecond laser pulse. Journal of Applied Physics 2011, 109(2): 023503.

\section{ISSUE DATE:}

2011-01

URL:

http://hdl.handle.net/2433/138082

\section{RIGHT:}

(C) 2011 American Institute of Physics. 


\title{
Thermal and shock induced modification inside a silica glass by focused femtosecond laser pulse
}

\author{
Masaaki Sakakura, ${ }^{1, a)}$ Masahide Terazima, ${ }^{2}$ Yasuhiko Shimotsuma, ${ }^{1}$ Kiyotaka Miura, ${ }^{3}$ and \\ Kazuyuki Hirao ${ }^{3}$ \\ ${ }_{1}^{1}$ Kyoto University Office of Society-Academia Collaboration for Innovation, Kyoto 615-8520, Japan \\ ${ }^{2}$ Department of Chemistry, Graduate School of Science, Kyoto University, Kyoto 606-8502, Japan \\ ${ }^{3}$ Department of Material Chemistry, Graduate School of Engineering, Kyoto University, \\ Kyoto 615-8510, Japan
}

(Received 4 October 2010; accepted 1 December 2010; published online 18 January 2011)

The femtosecond laser induced structural modification was studied by the detection of a laser induced pressure wave using a transient lens (TrL) method. The TrL signals observed at various excitation pulse energies showed that there were two thresholds of the pulse energy for the modification. Above the higher threshold, a pressure wave was observed clearly, and the amplitude of the pressure wave increased with increasing excitation pulse energy. In addition, Raman spectra at the laser irradiated region showed that the compact silica ring structures increased in the photoexcited region above the higher threshold, which suggested that the photoexcited glass was densified by a shock due to a pressure wave generation. In the energy region between the lower and higher thresholds, a pressure wave was not detected but a temporally constant refractive index change was observed. From the amplitude of the change, the temperature elevation just after the photoexcitation in this energy region was estimated to be about $1400 \mathrm{~K}$. We interpreted that fast cooling from the high temperature is responsible for the modification between the lower and higher thresholds. The two modifications identified in this study should correspond to two types of damage inside a silica glass which have been observed previously. This is the first study that elucidated the difference of two types of modification from the time-resolved observation of the dynamics. (0) 2011 American Institute of Physics. [doi:10.1063/1.3533431]

\section{INTRODUCTION}

Structural modifications inside glasses induced by femtosecond (fs) laser irradiation have been investigated by a number of researchers. ${ }^{1-19}$ In particular, the modifications accompanied with large refractive index change are important, because they can be applied to the fabrication of various optical devices, such as waveguides, ${ }^{1,2,8,9,18}$ gratings, ${ }^{15,16}$ or photonic crystals. ${ }^{17}$ When the induced refractive index change is positive, ${ }^{1,2,9,18}$ a line of the refractive index change works as an optical waveguide.

This laser processing has often been applied to fabrication of various three-dimensional optical devices inside a silica glass, because this glass is an important material in the optical communication because of the low optical loss in a wavelength range. ${ }^{20}$ Therefore, there have been number of investigations on the fs laser induced modification inside a silica glass. ${ }^{5,8-13,18,19}$ For example, Chan et al. ${ }^{9}$ observed the Raman spectrum from the region of the refractive index change inside a silica glass and found that the smaller rings of $\mathrm{SiO}_{2}$ structure had increased. Their results suggested that densification occurred after the photoexcitation by fs laser pulses. They speculated that the densification had been induced by fast cooling after the photoexcitation or shock wave generation. ${ }^{10}$ Mysyrowicz and co-workers ${ }^{18}$ found two types of damages in the fs laser focal region inside a silica glass. They compared the spatial dynamics of excited elec-

${ }^{a)}$ Electronic mail: msakakura@saci.kyoto-u.ac.jp. trons with the damages and suggested that the damage with larger pulse energy (type II damage) should be associated with avalanche ionization. They also suggested that a molten phase of $\mathrm{SiO}_{2}$ should be formed due to the high temperature after the photoexcitation based on a result from the timeresolved light transmission measurement. In spite of these efforts, the origin of the refractive index change as well as the difference in the mechanism between two types of damages have not been elucidated well.

One of the possible origins of the fs laser induced refractive index change is a pressure wave, which is generated as a result of the rapid relaxation of thermoelastic stress. ${ }^{5,6,18} \mathrm{Be}-$ cause a pressure wave compresses materials, the refractive index increase in a fs laser focal region might be the result of the densification by the pressure wave. Another possible origin is a rapid temperature elevation and rapid cooling. ${ }^{7}$ A number of studies showed that rapid cooling of a silica glass induces a glass structure of a higher fictive temperature, which has a higher density. ${ }^{21,22}$ However, there have been little studies on the relation between the laser induced refractive index change, pressure wave, and temperature change.

For investigating the relation between the fs laser induced modification and pressure wave, the observation of the pressure wave is essential. In our previous studies, we showed that the fs induced pressure wave inside a glass can be detected with a high sensitivity by the transient lens (TrL) method, which can detect the laser induced refractive index change with high sensitivity. ${ }^{5-7}$ We successfully observed a pressure wave as a damping oscillation in the TrL signal. ${ }^{6}$ 
Since the amplitude of the oscillation in a TrL signal represents the amplitude of the pressure wave, this method is suitable for detecting the pressure wave in the fs laser processing.

In this study, we observed the TrL signals from the fs laser focused area in a silica glass at various excitation laser pulse energies, and investigated the relation between the laser induced modification and the pressure wave generation. We found that there are two thresholds of the pulse energy for the structural modifications. In an excitation energy region above the higher threshold, a compact silica network, which was detected with micro-Raman spectroscopy, appeared and the amplitude of the pressure wave increased with increasing laser pulse energy.

In the excitation energy region between the lower and higher thresholds, a pressure wave was too small to be detected and a smooth refractive index line can be drawn. Based on the temperature increase and pressure increase, which were evaluated from the TrL signals, we discussed the mechanism responsible for two types of structural modifications.

\section{PRINCIPLE}

\section{A. Detection of laser induced pressure wave by a TrL method}

The TrL method is one of the methods to observe the dynamics of the photoinduced refractive index change. ${ }^{5-7,23-26}$ Because a laser induced pressure wave alters refractive index distribution around the photoexcited region, it can be observed by the TrL method. The principle of the TrL method is shown schematically in Fig. 1(a). When a probe beam passes through the photoexcited region, the wavefront is deformed by the refractive index distribution, which is induced by the photoexcitation. The wavefront deformation results in the modulation of the spatial intensity distribution at the far field due to the diffraction. ${ }^{27}$ Because the modulation of the intensity distribution comes from the refractive index distribution, the refractive index dynamics can be observed by detecting the temporal evolution of the intensity distribution. In the TrL method, the light intensity at the center of the probe beam is detected. The temporal evolution of the intensity is called a "TrL signal."

A variety of dynamics accompanied with refractive index change can be observed by the TrL method, for example, optical Kerr effect, plasma formation, change of chemical species, density change, and so on. ${ }^{7,23-26}$ In particular, the fs laser induced pressure wave generates a characteristic $\mathrm{TrL}$ signal. ${ }^{5,6}$ The typical TrL signals of a fs-laser induced pressure wave are shown in Figs. 1(b) and 1(c), which were obtained by an experiment and calculation, respectively. In both the experimental and calculated TrL signals, the damping oscillation appears. In the previous paper, we showed that the damping oscillation comes from the propagation of a fs-laser induced pressure wave inside a glass. ${ }^{6}$ We called this oscillation "TrL oscillation." The TrL oscillation is a good indicator of the generation of a pressure wave, because the amplitude of the TrL oscillation increases as that of the pressure wave becomes larger ${ }^{6}$ and this method has a high sen- (a)

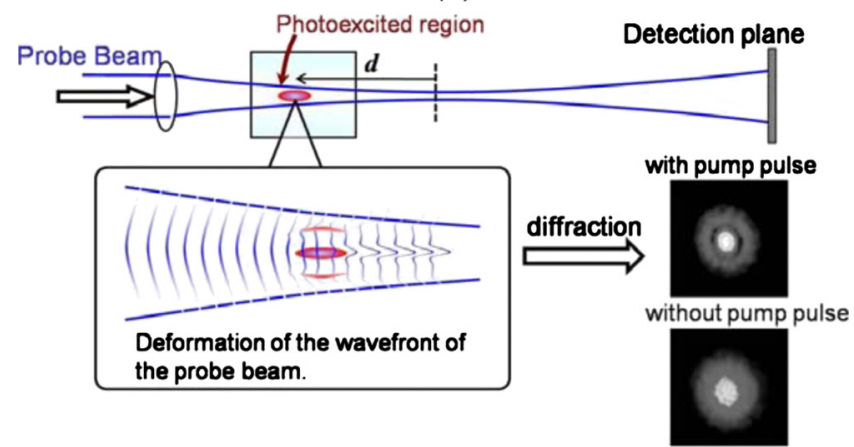

(b)

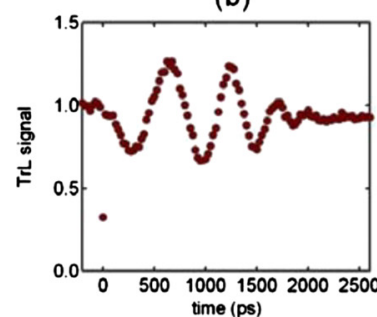

time (ps)

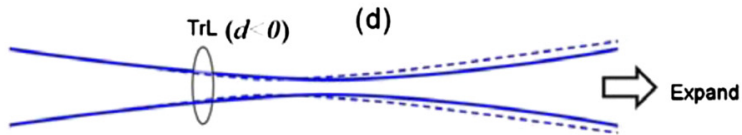

$\operatorname{TrL}(d>0)$

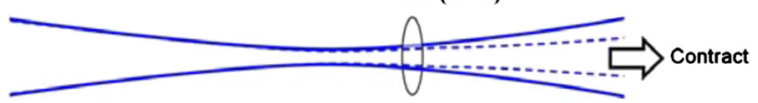

FIG. 1. (Color online) (a) Principle of the TrL method. (b) A typical TrL signal when a pressure wave was generated after photoexcitation inside a glass by fs laser pulse. (c) A TrL signal which was simulated by a phase distribution modulated by a pressure wave propagation. (d) Probe beam propagations after passing a convex TrL at positive (upper) or negative (below) focal mismatch $(d)$. The broken and solid lines indicate the beam propagation with and without the TrL, respectively.

sitivity. In this study, we measured the amplitude of the TrL as a function of excitation pulse energy, and investigated the relation between the amplitude and the structural change.

\section{B. Focal mismatch and the sign of a TrL signal}

The focal mismatch between the excitation and probe beams, which is denoted by $d$, is important for analyzing TrL signals, because the TrL signal depends on this value. ${ }^{5}$ In this study, we defined that $d$ is positive when the probe beam is focused before passing through the photoexcited region. For example, $d$ is negative in the case of Fig. 1(a), because the photoexcited region is before the focal position of the probe beam. The sign of $d$ determines whether the TrL signal increases or decreases. For example, we consider the TrL with a positive Gaussian shaped-refractive index distribution [Fig. 1(d)]. This TrL works as a convex lens. As shown in Fig. 1(d), the convex TrL at negative $d$ moves the focal position of the probe beam backward. This movement results in the expansion of the probe beam at the far field. Therefore, the TrL signal intensity decreases in this case. On the other hand, a convex $\operatorname{TrL}$ at a positive $d$ contracts the probe beam, which results in the increase in $\operatorname{TrL}$ signal. Therefore, we can determine the sign of the refractive index change from the TrL 


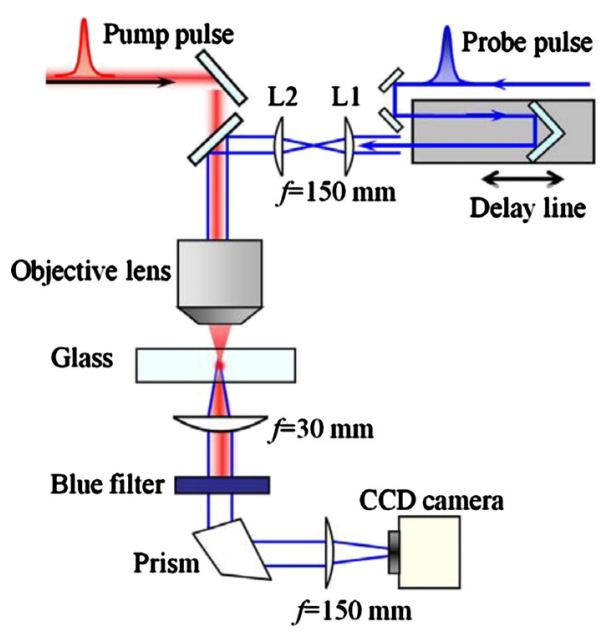

FIG. 2. (Color online) Experimental setup for the TrL method.

signal if $d$ is known. In addition, we can determine whether the signal intensity change comes from refractive index change or light absorption by observing TrL signals at different $d$, because light absorption decreases the signal intensity at any $d$.

\section{EXPERIMENTAL}

\section{A. TrL method}

The experimental setup for the TrL detection has been described in other articles (Fig. 2) ${ }^{5,6}$ In short, near-IR fs laser pulse (Coherent Inc.; Mira9000-Legend, the repetition rate was $1 \mathrm{kHz}$, the center of the wavelength was $800 \mathrm{~nm}$, and the pulse duration was $120 \mathrm{fs}$ ) was focused inside a silica glass plate (ShinEtsu QUARTS; VIOSIL-SQ) with an optical microscope objective $(20 \times, \mathrm{NA}=0.45)$, and the photoexcitation was induced in the laser focal region. The focal position of the excitation pulse was about $300 \mu \mathrm{m}$ depth from the glass surface. The probe laser pulse $\left(\lambda_{\text {probe }}=400 \mathrm{~nm}, 120 \mathrm{fs}\right)$, which was frequency doubled replica of the excitation pulse by a $\beta-\mathrm{BaB}_{2} \mathrm{O}_{4}(\mathrm{BBO})$ crystal and then optically delayed, passed through the photoexcited region coaxially with the excitation beam. After the probe beam transmitted through the photoexcited region, the intensity distribution at about 30 $\mathrm{mm}$ apart from the photoexcited region was imaged on an intensified charge coupled device camera (HAMAMATSU; C10054-03). Before the detection, the excitation beam was attenuated by a blue filter and eliminated completely from the probe beam by a prism. The TrL signal was obtained by plotting the intensities at the center of the probe beams against the delay times of the probe pulses. The pulse energy was controlled by an optical neutral density filter. The glass plate was translated at about $10 \mathrm{~mm} / \mathrm{s}$ during the data acquisition to avoid multiexcitation at the same area. The focal mismatch $(d)$ was controlled by changing the divergence of the probe beam with a telescope of two lenses (L1 and L2).

\section{B. Analysis of refractive index lines}

The optical setup for writing refractive index lines by focusing fs laser pulses inside a silica glass was the same as that of the TrL method without a probe beam. In writing (a)
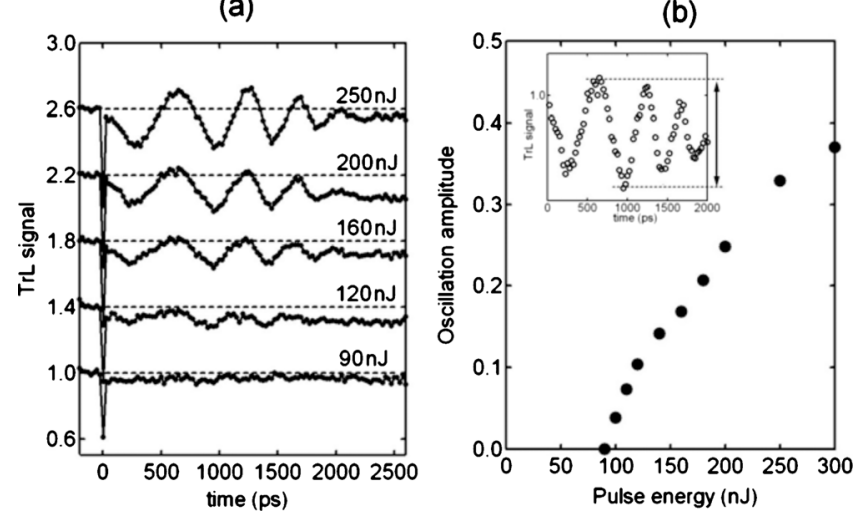

FIG. 3. (a) TrL signals at $d=-0.06 \mathrm{~mm}$ with various excitation energy. The signals are offset for clarity and the baselines of the signals are drawn by broken lines. (b) Plot of the oscillation amplitude against the excitation energy (opened circles). The inset shows the definition of the oscillation amplitude, which is defined by the difference between the signal intensity at the first positive peak and that at the second negative peak.

refractive index lines, the glass plate was translated at $100 \mu \mathrm{m} / \mathrm{s}$, at which the adjacent laser induced structures were overlapped. The morphologies of the refractive index lines were observed with an optical microscope with a $50 \times$ objective lens (NIKON; LU Plan). The Raman spectra in the refractive index lines were measured with a confocal laser scanning microscope (Tokyo Instruments, Nanofinder 30). The excitation laser beam from a laser diode-pumped $\mathrm{Nd}$ :YAG laser with a wavelength of $532 \mathrm{~nm}$ was focused in the refractive index line with a $40 \times$ objective lens and the spectra of the Raman scattering were analyzed with a spectrometer.

\section{RESULTS}

\section{A. Excitation pulse energy dependence of TrL signals}

The TrL signals at various excitation pulse energies $\left(I_{e x}\right)$ at $d=-0.06 \mathrm{~mm}$ are shown in Fig. 3(a). In all the TrL signals, spike shaped signals at the delay time of 0 ps were observed as the decrease of the probe light intensity. This spike signal should be attributed to an optical Kerr effect and plasma formation, which has been reported by other researchers. ${ }^{5,6,23,28}$ After this signal, the TrL oscillations were clearly observed at the strong excitation pulse energy. The amplitude of the TrL oscillation became larger with increasing the energy. Previously, we showed that this oscillation represents the pressure wave generated by the excitation pulse. $^{6}$ This oscillation disappeared at $I_{e x}<90 \mathrm{~nJ}$. This means that the amplitude of a pressure wave is negligibly small at $I_{e x}<90 \mathrm{~nJ}$. To obtain the energy threshold of the generation of a pressure wave, the oscillation amplitudes were plotted against $I_{e x}$ in Fig. 3(b). From this plot, we determined the energy threshold of the pressure wave generation to be $90 \mathrm{~nJ}$.

Figure 4(a) shows the excitation energy dependence of the TrL signal below the energy threshold of a pressure wave generation $\left(I_{e x}=<90 \mathrm{~nJ}=I_{t h-H}\right)$. It is interesting to note that the TrL signal after the first spike did not recover to the baseline (light intensity before the photoexcitation) and a temporally constant negative component was observed. This 
(a)

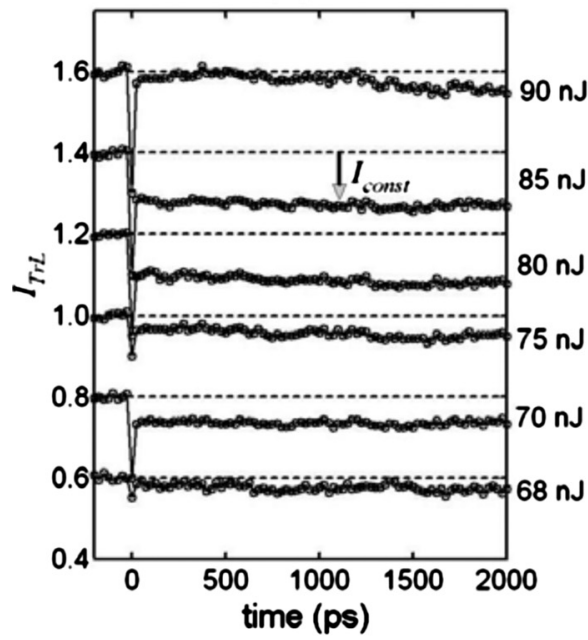

(b)

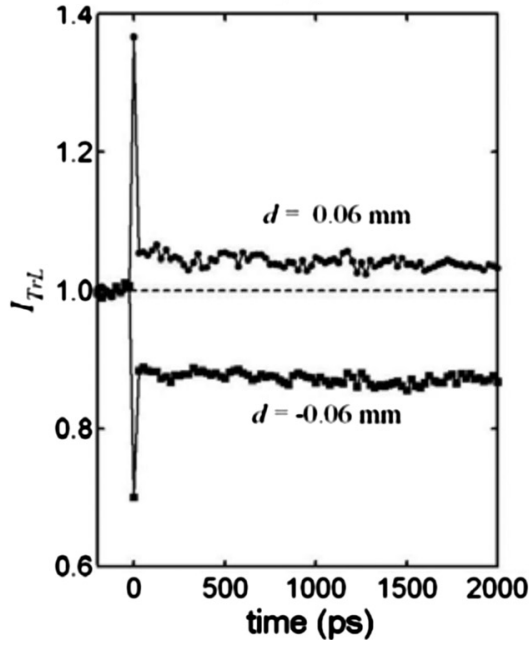

(c)

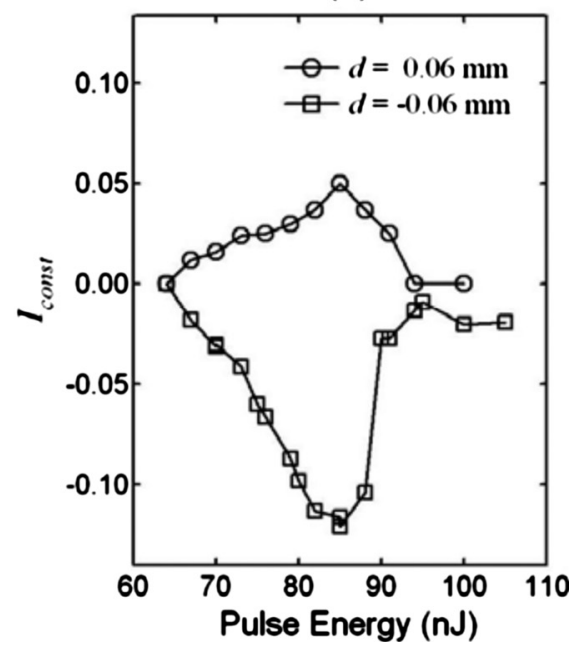

FIG. 4. (a) TrL signals below the energy threshold of a pressure wave generation. (b) TrL signals of the excitation pulse energy of $85 \mathrm{~nJ}$ at $d=0.06$ and -0.06 $\mathrm{mm}$. (c) The intensity of the temporally constant signal $\left(I_{\text {const }}\right)$ at different $d$ s plotted against the pulse energies.

signal clearly indicated that a structural modification was induced even by this weak photoexcitation in which a pressure wave was too small to be observed. There are two possibilities for the origin of the temporally constant negative component; one is light absorption by photoexcited material and the other is a refractive index lens effect. To identify the origin, the TrL signals were measured at negative and positive focal mismatches $(d)$. The TrL signals of $I_{e x}=85 \mathrm{~nJ}$ at $d=+0.06$ and $-0.06 \mathrm{~mm}$ are shown in Fig. 4(b). The temporally constant component was negative at a negative $d$ while it was positive at a positive $d$. This $d$-dependence means that a convex lens was created in the photoexcited region just after the photoexcitation, i.e., the refractive index increased [Fig. 1(d) case]. Figure 4(c) shows the plot of the signal intensity ( $\left.I_{\text {const }}\right)$ against $I_{e x}$. At the pulse energies in $I_{e x}$ $<I_{t h-H}$, the signs of the temporally constant signals at $d=$ $+0.06 \mathrm{~mm}$ and $-0.06 \mathrm{~mm}$ were positive and negative, respectively. According to the above explanation on the $d$-dependence, this means that the refractive index increased by the photoexcitation in this energy range. The temporally constant signal intensity became the largest at $I_{e x}=85 \mathrm{~nJ}$ and undetectable below $65 \mathrm{~nJ}\left(=I_{t h-L}\right)$.

From the TrL oscillation at stronger excitation and the temporally constant signal at weaker excitation in the $\operatorname{TrL}$ signals, there should be two thresholds, $I_{t h-H}$ and $I_{t h-L}$; above $I_{t h-H}$, the amplitude of the pressure wave is sensitive to the change in excitation pulse energy, and between $I_{t h-H}$ and $I_{t h-L}$, the pressure wave is undetectable but the refractive index in the photoexcited region increases after the photoexcitation.

\section{B. Morphologies of the refractive index lines}

Figure 5 shows the refractive index lines which were written inside a silica glass by focusing fs-laser pulses with a $20 \times$ objective lens. At the excitation pulse energy above $I_{t h-H}$, the refractive index lines were not smooth. On the other hand, the refractive index lines look smooth and continuous below $I_{t h-H}$. This difference suggests that the strain due to the pressure wave generation could have some influence to writing a refractive index line in a silica glass.

The cross sections of the refractive index lines are shown in Figs. 5(b) and 5(c). The longitudinal lengths of the modifications were about $30 \mu \mathrm{m}$ for $I_{e x}=300 \mathrm{~nJ}$ and $8 \mu \mathrm{m}$ for $I_{e x}=85 \mathrm{~nJ}$. The length for $I_{e x}=85 \mathrm{~nJ}$ was nearly as short as the Rayleigh length while that for $I_{e x}=300 \mathrm{~nJ}$ was about three times longer than the Rayleigh length. This elongation should be attributed to the filamentation of the laser pulse due to higher energy, which is the result of the refractive index distribution induced by plasma formation, optical Kerr effect, and other nonlinear optical effects ${ }^{11,12,18,19,28,29}$

\section{Raman spectra of laser focused region}

The Raman spectra from the center of the refractive index lines with various pulse energies are shown in Fig. 6(a). (a)

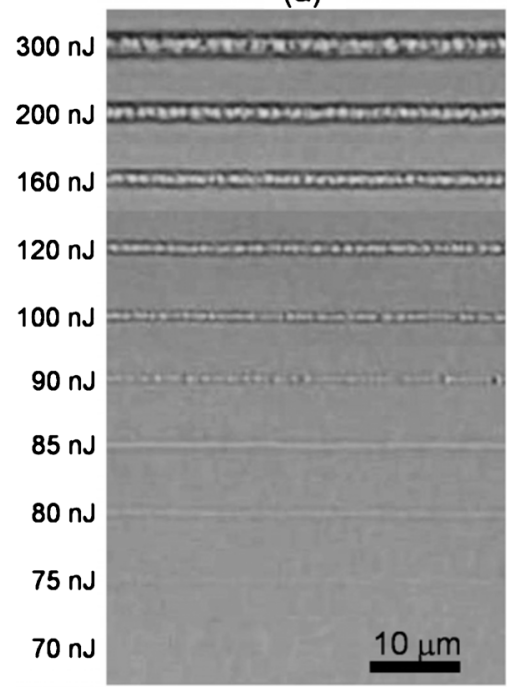

(b)

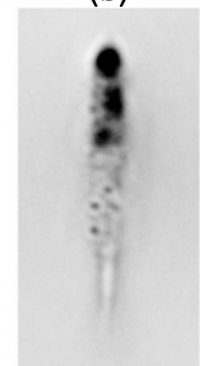

(c)

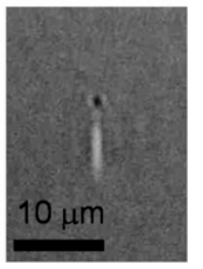

FIG. 5. (a) Optical microscope images of the refractive index lines written by fs laser irradiation inside a silica glass. [(b) and (c)] The cross section of the refractive index lines of the pulse energies of $300 \mathrm{~nJ}$ and $85 \mathrm{~nJ}$, respectively. 
(a)
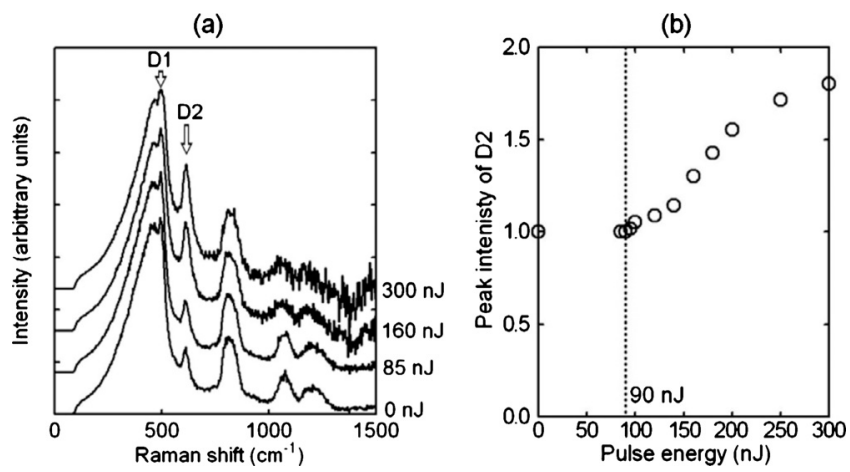

FIG. 6. (a) Raman spectra at the center of the refractive index lines written with various pulse energies. (b) The peak intensities of the D2 bands plotted against the pulse energies. The intensities were normalized by that from the nonirradiated area.

For clarity, all the Raman spectra after subtracting a luminescence from laser induced defects around $650 \mathrm{~nm},{ }^{30}$ and then corrected for temperature and frequency dependence ${ }^{31}$ of Raman scattering are shown. All the Raman bands observed in Fig. 6(a) have been assigned. ${ }^{32,33}$ For example, the dominant broadband around $450 \mathrm{~cm}^{-1}$ has been attributed to symmetric oxygen vibration of the bent $\mathrm{Si}-\mathrm{O}-\mathrm{Si}$ linkages. The sharp bands at $\sim 490 \mathrm{~cm}^{-1}$ (D1 band) and $\sim 600 \mathrm{~cm}^{-1}$ (D2 band) have been attributed to the oxygen breathing modes in fourand three-membered rings of $\mathrm{SiO}_{4}$ tetrahedra, respectively. These bands are important for obtaining the structural information in the laser induced modification inside a silica glass, because these bands come from the compaction of silica networks. ${ }^{32}$ Here, we focused the intensity change in the D2 band, because this band can be separated more easily from the other bands. The intensity of the D2 band increased with increasing laser pulse energy. The peak intensities of this band were plotted against the laser pulse energies in Fig. 6(b). The peak intensity of this band started to increase at about 90 nJ. From the comparison of the pulse energy dependences of the TrL oscillation and a D2 band [Figs. 3(b) and 6(b)], the threshold of a pressure wave $\left(I_{t h-H}\right)$ is nearly equal to the threshold of an increase in three-membered rings of $\mathrm{SiO}_{4}$ tetrahedra. This suggests that the three-membered ring structure in silica networks should be induced by a pressure wave generation. The excitation energy dependence of the D1 band, another indicator of compact structure in silica networks, was much smaller. This point will be discussed later.

\section{DISCUSSION}

We found that there should be two types of structural modification; one above the higher threshold is accompanied with a pressure wave generation and the other between the lower and higher thresholds without a pressure wave generation. First, we discuss the modification accompanied with a pressure wave generation. According to the simulation of elastic deformation, the pressure wave is generated as the result of the stress relaxation in the photoexcited region. ${ }^{5,34}$ After the photoexcitation, the temperature in the photoexcited region is elevated due to the energy transfer from the photoexcited electrons to the lattice in a glass, $5,18,19,28,29,34,35$ (a)
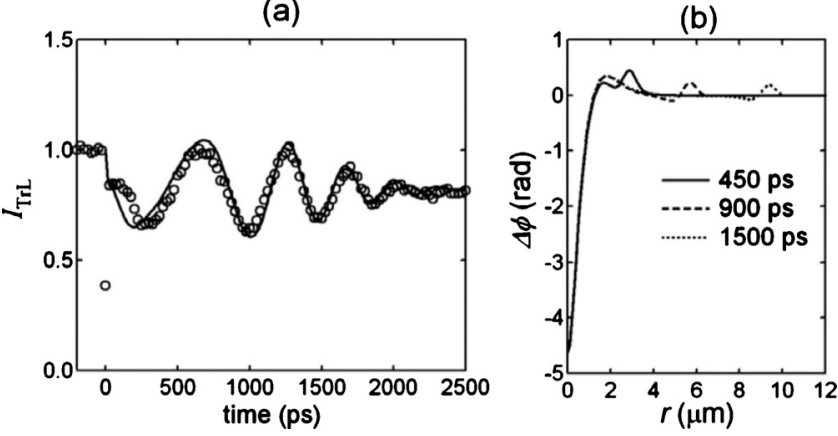

FIG. 7. (a) TrL signal of a silica glass at $I_{e x}=300 \mathrm{~nJ}$ (open circles) and the fitting line (a solid line), which was calculated using a time-dependent phase distribution of a probe beam shown in (b). (b) The phase distributions at 450, 900, and $1500 \mathrm{ps}$, which were used to simulate the fitting line in (a). The phase distributions include the contributions from a laser induced pressure wave and the refractive index change in the central region.

and the temperature elevation induces thermoelastic stress in the photoexcited region. Because the temperature elevation by fs laser irradiation occurs much faster than the elastic deformation of the material, ${ }^{28}$ the thermoelastic stress reaches the maximum just after the photoexcitation. The subsequent stress relaxation induces the generation of a pressure wave. Because this study showed that the threshold of a pressure wave was nearly equal to that the increase in the D2 band in the Raman spectrum, which reflects a compact structure in a silica glass, ${ }^{32}$ the densification could be generated by the shock due to the instantly generated thermoelastic stress in the photoexcitation.

The amplitude of the shock (i.e., pressure) due to the pressure wave can be estimated from the TrL signal. First, the TrL signal was fitted by the phase distribution which was calculated based on the simulated pressure wave. The fitted TrL signal at $I_{e x}=300 \mathrm{~nJ}$ and the time-dependent phase distributions are shown in Figs. 7(a) and 7(b), respectively. In the process of fitting the TrL signal, the phase change and the width of the pressure wave were changed as parameters to reproduce the observed TrL signal. From the phase distributions shown in Fig. 7(b), the largest phase shift due to the pressure wave was $\Delta \phi=0.4 \mathrm{rad}$. Next, the refractive index change was obtained using $\Delta \phi=0.4 \mathrm{rad}$. The relation between the refractive index change $\Delta n$ and the phase shift $\Delta \phi$ is given by

$$
\Delta \phi=2 \pi \frac{\Delta n l}{\lambda_{\text {probe }}},
$$

where $l$ is the longitudinal length of the photoexcited region. According to the cross section of the refractive index line at $I_{e x}=300 \mathrm{~nJ}$ [Fig. 5(b)], the longitudinal length was assumed to be $l=30 \mu \mathrm{m}$. Therefore, the refractive index change due to the pressure wave was $\Delta n=8.5 \times 10^{-4}$. The refractive index change in a condensed matter is related to the density change by the Lorenz-Lorentz relation, ${ }^{36}$

$$
\Delta n_{\rho}=\frac{n_{0}{ }^{4}-1}{4 n_{0}}\left(\frac{\Delta \rho}{\rho_{0}}\right),
$$

where $\rho_{0}$ and $n_{0}$ are, respectively, the density and refractive index of a silica glass before the densification. The combina- 
tion of Eqs. (1) and (2) gives the following relation between the density change and phase shift:

$$
\frac{\Delta \rho}{\rho_{0}}=\Delta \phi \frac{\lambda_{\text {probe }}}{2 \pi l} \frac{4 n_{0}}{n_{0}^{4}-1} .
$$

Using the refractive index of a silica glass at $\lambda_{\text {probe }}$ $=400 \mathrm{~nm} \quad\left(n_{0}=1.47\right)$, the density change to induce $\Delta \phi$ $=0.4 \mathrm{rad}$ was calculated to be about $0.14 \%$. Because the stress due to density change is given by ${ }^{37}$

$$
\Delta P=K\left(\frac{\Delta \rho}{\rho_{0}}\right)
$$

where $K$ is the bulk modulus of a silica glass $(=37 \mathrm{GPa}$ at $293 \mathrm{~K}$ ), the maximum stress due to the pressure wave was 50 $\mathrm{MPa}$ at $I_{e x}=300 \mathrm{~nJ}$. According to the thermoelastic simulation, the initial stress in the photoexcited region which induces a pressure wave of $50 \mathrm{MPa}$ must be $520 \mathrm{MPa}$, which is generated by the thermal expansion of $1.4 \%$. It has been reported that the shock pressure which induces the apparent increase in D2 band must be higher than several gigapascals $(\mathrm{GPa}){ }^{33}$ The estimated stress due to fs laser irradiation, 520 $\mathrm{MPa}$, is lower than the reported value. This suggests that not only pressure increase but also temperature elevation should be necessary for the densification of a silica glass after fslaser irradiation. The temperature elevation was estimated to be $23000 \mathrm{~K}$, based on the estimated density change and the thermal expansion coefficient of a silica glass $(=0.62$ $\left.\times 10^{-6}\right){ }^{38}$ This temperature elevation must be overestimated, because this estimation is based on the assumption that the thermal expansion coefficient is independent of temperature. In spite of the overestimation, we can expect that the temperature after the photoexcitation should be much higher than the melting point of a $\beta$-cristobalite $\left(\sim 1730{ }^{\circ} \mathrm{C}\right) .^{39}$ Therefore, not only the high pressure but also the temperature beyond the melting point should play an important role in the fs-laser induced densification of a silica glass. The Raman spectra in this study showed that only the intensity of the D2 band was sensitive to the excitation energy. This means that the three-membered ring of silica network increased by high pressure under high temperature. On the other hand, it has been reported that both the intensities of the D1 and D2 bands increase in a silica glass densified by shock wave or high pressure above several GPa. This difference suggests that the mechanism of the densification in a silica glass by fs-laser irradiation should be completely different from those of shock induced densification and densification under high pressure.

Next, the modification between $I_{t h-H}$ and $I_{t h-L}$ is discussed. In this energy range, a temporally constant component was observed until 2 ns in a TrL signal. This component means that the refractive index increased just after the photoexcitation. There are four possible origins of the refractive index change. ${ }^{25,28,29}$ The refractive index after photoexcitation can be described by (a)
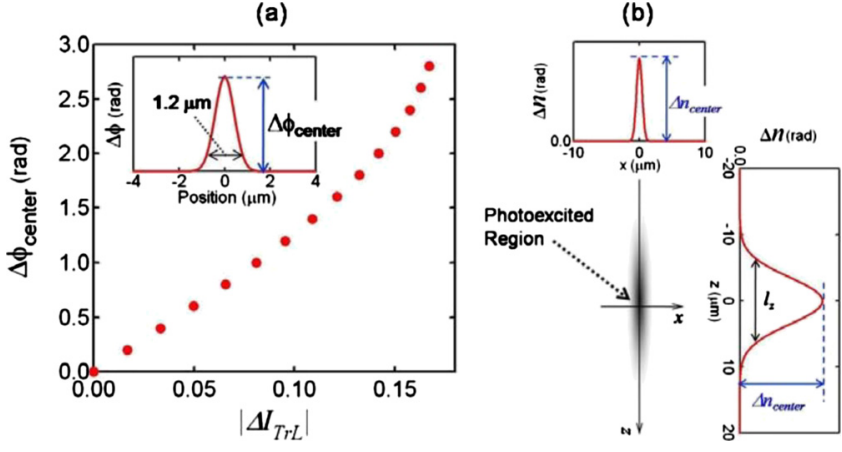

FIG. 8. (Color online) (a) The relation between the phase shift at the center and the TrL signal change, which was obtained by simulating a TrL signal by the Gaussian shaped phase distribution whose width is $1.2 \mu \mathrm{m}$. The phase shift at the center is defined in the inset. (b) Simplified refractive index distribution just after the photoexcitation in the photoexcited region. Because the probe beam propagates along the $\mathrm{z}$ axis, the phase shift can be obtained by integrating the refractive index along the $\mathrm{z}$ axis.

$$
\Delta n=\left(\frac{\partial n}{\partial T}\right)_{\rho} d T+\left(\frac{\partial n}{\partial \rho}\right)_{T} d \rho+\Delta n_{\text {plasma }}+\Delta n_{\text {trap }}
$$

where the first term is the refractive index change due to temperature change, the second one is that due to density change, the third one is that due to photoinduced plasma, and the forth one is that of the photoinduced defect. The photoinduced plasma cannot explain the positive refractive index change, since it contributes negatively to the refractive index. ${ }^{28,29}$ In addition, the contribution by the density change is negligible, since a pressure wave generation (TrL oscillation) was not detected below $I_{t h-H}$. On the other hand, the refractive index increases with increasing temperature at constant density, ${ }^{38,40}$ and the refractive index change due to photoinduced defect is positive if the frequency of the light absorption by the defect is higher than that of the probe beam. Therefore, we can conclude that the temporally constant refractive index change below $I_{t h-H}$ comes from temperature elevation or photoinduced defect.

The maximum value of possible temperature change can be estimated by neglecting the contribution by the photoinduced defect and the temperature coefficient of the refractive index of a silica glass. The temporally constant component in the $\operatorname{TrL}$ signal at $I_{e x}=85 \mathrm{~nJ}$ was $-0.12\left(=\Delta I_{\mathrm{TrL}}\right)$ at $d=$ $-0.06 \mathrm{~mm}$. We simulated the TrL signal intensity by the Gaussian shaped refractive index change. In this simulation, we assumed that the width of the refractive index distribution was $1.2 \mu \mathrm{m}$ [the inset of Fig. 8(a)], which is the same as the width of the refractive index line at $I_{e x}=85 \mathrm{~nJ}$ (Fig. 5). The relation between the signal intensity change $\Delta I_{\mathrm{TrL}}$ and the phase shift of the probe beam at the center $\left(\Delta \phi_{\text {center }}\right)$ was shown in Fig. 8(a). From this relation, the phase shift at the center was determined to be $\Delta \phi_{\text {center }} \sim 1.5 \mathrm{rad}$. The relation between the phase shift and the refractive index was obtained based on the model shown in Fig. 8(b). $\Delta \phi_{\text {center }}$ can be obtained by integrating the refractive index along the beam axis: 


$$
\begin{aligned}
\Delta \phi_{\text {center }} & =2 \pi \int_{-\infty}^{\infty} \frac{\Delta n_{\text {center }}}{\lambda_{\text {probe }}} \exp \left[-\left(\frac{z}{l_{z} / 2}\right)^{2}\right] d z \\
& =\pi^{3 / 2} \frac{\Delta n_{\text {center }}}{\lambda_{\text {probe }}} l_{z}
\end{aligned}
$$

where $l_{z}$ is the longitudinal length of the temperature distribution. If $l_{z}$ is estimated from the cross section of the refractive index line shown in Fig. 5(c), i.e., $l_{z} \sim 8.0 \mu \mathrm{m}$, the calculated refractive index change at the center is $\Delta n_{\text {center }}=1.3$ $\times 10^{-2}$. Using the temperature coefficient of the refractive index $\left(d \mathrm{n} / d \mathrm{~T}=9.5 \times 10^{-6} \mathrm{~K}^{-1}\right.$ at $\left.400 \mathrm{~nm}\right),{ }^{40}$ the temperature elevation at the center was calculated to be about $1400 \mathrm{~K}$, i.e., the temperature at the center just after the photoexcitation was $1425^{\circ} \mathrm{C}$. This estimation suggests that the glass in the photoexcited region was heated above the glass transition range $\left(1150-1300{ }^{\circ} \mathrm{C}\right)$, at which the glass network can be relaxed to the stable structure under high temperature. ${ }^{41}$ After the relaxation of the glass network, the subsequent quenching could freeze the equilibrium structure at high temperature, which results in the refractive index change. At the estimated temperature at the center $\left(1425^{\circ} \mathrm{C}\right)$, the viscosity of a silica glass should be $\eta \sim 10^{8.6} \mathrm{~Pa} \mathrm{~s}^{42}$ Because the viscoelastic relaxation time ${ }^{43}$ is given by $\eta / G$, where $G$ is bulk modulus $\left(G=30-60 \mathrm{GPa}\right.$ at $0-2000{ }^{\circ} \mathrm{C}$ and under $0-5$ $\mathrm{GPa}),{ }^{44}$ the time needed for the glass to return the original structure is about $10 \mathrm{~ms}$, On the other hand, the cooling time after the fs laser focusing inside a glass is faster than $10 \mu \mathrm{s}$ according to our previous study. ${ }^{7}$ This cooling time is fast enough for the structure at high temperature to be fixed during the cooling process. Therefore, we conclude that the modification of the lower threshold should be generated by the fast quenching from the temperature near glass transition range.

Other researchers also found two types of structural change inside a silica glass after fs laser irradiation; type I appears for excitation pulse energies between lower and higher thresholds, and type II, above higher one. ${ }^{9,18}$ Mysyrowicz and co-workers interpreted that avalanche ionization occurred in type II damage while not in type I one based on the simulation of photoexcitation of electrons. In the avalanche ionization, multiplication of photoexcited electrons by electromagnetic field of a laser pulse generates high density plasma and the plasma absorbs light energy strongly; therefore, the light absorption is sensitive to the excitation pulse energy at which avalanche ionization occurs. Our present measurement revealed that the amplitude of a pressure wave was sensitive to the laser intensity in the structural change above $I_{t h-H}$ while it was not sensitive in that between lower and higher thresholds. This suggests that the modification above $I_{t h-H}$ should correspond to the type II damage. In addition, Mysyrowicz and co-workers found that type I damage disappeared by heating a sample above $900{ }^{\circ} \mathrm{C}$. This fact indicates that the type I damage should be the result of fast quenching of a silica glass from the non-equilibrium state above the glass transition range. As discussed above, we concluded that the modification below $I_{t h-H}$ should be generated by fast quenching. Therefore, the modification below $I_{t h-H}$ should correspond to the type I damage. Because a pressure wave was not detected in the modification below $I_{t h-H}$, we can interpret that a strong pressure wave in the type II damage should generate irreversible structural change and prevent the photoexcited material from returning the original state by heating.

\section{CONCLUSION}

In conclusion, we found two thresholds of the fs laser induced modification inside a silica glass based on the observation of refractive index dynamics by the TrL method. The modification above the higher threshold was accompanied with the generation of a pressure wave after the photoexcitation. We consider that the pressure wave is the main origin of the structural change in the glass. On the other hand, below the lower threshold, a pressure wave was too small to be observed, although there was a temporally constant component in the TrL signal. Regardless of undetectable pressure wave, the temperature at the photoexcited region, which was estimated from the TrL signal intensity, was above the glass transition range. Considering both the temperature and the fast cooling rate, we interpreted that the fast cooling from the structure under high temperature should be responsible to the modification between the lower and higher thresholds. Two types of modification have also been observed by other researchers. This study clarified the two types of modification from the observation of the dynamics for the first time.

\section{ACKNOWLEDGMENTS}

The authors thank Professor Qiu from Zhejiang University, Professor Kazansky from University of Southampton, and Dr. Kanehira and Dr. Nishi from Kyoto University for good suggestion and discussions. This research was partly supported by New Energy and Industrial Technology Development Organization (NEDO), the Murata science foundation, and Amada foundation for metal work technology.

${ }^{1}$ K. M. Davis, K. Miura, N. Sugimoto, and K. Hirao, Opt. Lett. 21, 1729 (1996).

${ }^{2}$ K. Miura, J. Qiu, H. Inouye, T. Mitsuyu, and K. Hirao, Appl. Phys. Lett. 71, 3329 (1997).

${ }^{3}$ H. Zhang, S. Eaton, and P. R. Herman, Opt. Express 14, 4826 (2006).

${ }^{4}$ E. N. Glezer, M. Milosavljevic, L. Huang, R. J. Finlay, T.-H. Her, J. P. Callan, and E. Mazur, Opt. Lett. 21, 2023 (1996).

${ }^{5}$ M. Sakakura and M. Terazima, Phys. Rev. B 71, 024113 (2005).

${ }^{6}$ M. Sakakura, M. Terazima, Y. Shimotsuma, K. Miura, and K. Hirao, Opt. Express 15, 5674 (2007)

${ }^{7}$ M. Sakakura, M. Terazima, Y. Shimotsuma, K. Miura, and K. Hirao, Opt. Express 15, 16800 (2007).

${ }^{8}$ A. M. Streltsov and N. F. Borrelli, J. Opt. Soc. Am. B 19, 2496 (2002).

${ }^{9}$ J. W. Chan, T. R. Huser, S. H. Risbud, and D. M. Krol, Appl. Phys. A: Mater. Sci. Process. A76, 367 (2003).

${ }^{10}$ W. J. Reichman, J. W. Chan, C. W. Smelser, S. J. Mihailov, and D. M. Krol, J. Opt. Soc. Am. B 24, 1627 (2007).

${ }^{11}$ L. Sudrie, A. Couairon, M. Franco, B. Lamouroux, B. Prade, S. Tzortzakis, and A. Mysyrowicz, Phys. Rev. Lett. 89, 186601 (2002).

${ }^{12}$ A. Couairon and A. Mysyrowicz, Phys. Rep. 441, 47 (2007).

${ }^{13}$ L. Sudrie, M. Franco, B. Prade, and A. Mysyrowicz, Opt. Commun. 171, 279 (1999).

${ }^{14}$ V. Mizeikis, H.-B. Sun, A. Marcinkevičius, J. Nishii, S. Matsuo, S. Juodkazis, and H. Misawa, J. Photochem. Photobio., A 145, 41 (2001).

${ }^{15}$ Y. Shimotsuma, P. G. Kazansky, J. R. Qiu, and K. Hirao, Phys. Rev. Lett. 91, 247405 (2003).

${ }^{16}$ T. Toma, Y. Furuya, Y. W. Watanabe, K. Itoh, J. Nishii, and K. Hayashi, Opt. Rev. 7, 14 (2000) 
${ }^{17}$ M. Beresna and P. G. Kazansky, Opt. Lett. 35, 1662 (2010).

${ }^{18}$ A. Mermillod-Blondin, I. M. Burakov, Y. P. Meshcheryakov, N. M. Bulgakova, E. Audouard, A. Rosenfeld, A. Husakou, I. V. Hertel, and R. Stoian, Phys. Rev. B 77, 104205 (2008).

${ }^{19}$ A. Couairon, L. Sudrie, M. Franco, B. Prade, and A. Mysyrowicz, Phys. Rev. B 71, 125435 (2005).

${ }^{20}$ Y. P. Li and C. H. Henry, IEE Proc.: Optoelectron. 143, 263 (1996).

${ }^{21}$ R. Bruckner, J. Non-Cryst. Solids 5, 123 (1970).

${ }^{22}$ J. E. Shelby, J. Non-Cryst. Solids 349, 331 (2004).

${ }^{23}$ M. Terazima, Opt. Lett. 20, 25 (1995).

${ }^{24}$ M. Terazima, Chem. Phys. Lett. 230, 87 (1994).

${ }^{25}$ M. Terazima and N. Hirota, J. Chem. Phys. 100, 2481 (1994).

${ }^{26}$ J. F. Power, Appl. Opt. 29, 52 (1990).

${ }^{27}$ K. Iizuka, Engineering Optics (Springer-Verlag, Berlin, Tokyo, 1985), Chap. 2.

${ }^{28}$ P. Martin, S. Guizard, Ph. Daguzan, G. Petite, P. D’Oliveira, P. Meynadier, and M. Perdrix, Phys. Rev. B 55, 5799 (1997).

${ }^{29}$ S.-H. Cho, H. Kumagai, and K. Midorikawa, Opt. Commun. 207, 243 (2002).

${ }^{30}$ L. Skuja, J. Non-Cryst. Solids 167, 229 (1994).

${ }^{31}$ D. A. Long, Raman Spectroscopy (McGraw Hill, New York, 1977).

${ }^{32}$ P. F. McMillan, B. Piriou, and R. Couty, J. Chem. Phys. 81, 4234 (1984).
${ }^{33}$ M. Okuno, B. Reynard, M. Shimada, Y. Syono, and C. Willaime, Phys. Chem. Miner. 26, 304 (1999).

${ }^{34}$ A. Vogel, J. Noack, G. Huttman, and G. Paltauf, Appl. Phys. B: Lasers Opt. B81, 1015 (2005).

${ }^{35}$ S. W. Winkler, I. M. Burakov, R. Stoian, N. M. Bulgakova, A. Husakou, A. Mermillod-Blondin, A. Rosenfeld, D. A. Ashkenasi, and I. V. Hertel, Appl. Phys. A: Mater. Sci. Process. 84, 413 (2006).

${ }^{36}$ M. Born and E. Wolf, Principles of Optics (Pergamon, New York, 1980).

${ }^{37}$ L. D. Landau and E. M. Lifshitz, Theory of Elasticity (Pergamon, New York, 1986), Chap. 3.

${ }^{38}$ G. Ghosh, J. Non-Cryst. Solids 189, 191 (1995).

${ }^{39}$ F. E. Wagstaff, J. Am. Ceram. Soc. 52, 650 (1969).

${ }^{40}$ J. Matsuoka, N. Kitamura, S. Fujinaga, T. Kitaoka, and H. Yamashita, J. Non-Cryst. Solids 135, 86 (1991).

${ }^{41}$ N. V. Surovtsev, S. V. Adichtchev, V. K. Malinovsky, A. A. Kalininm, and Yu. N. Pal'yanov, J. Phys.: Condens. Matter 18, 4763 (2006).

${ }^{42}$ G. Urbain, Y. Bottinga, and P. Richet, Geochim. Cosmochim. Acta 46, 1061 (1982).

${ }^{43} \mathrm{~K}$. Varshneya, Fundamentals of Inorganic Glasses (Academic, New York, 1994), Chap. 13.

${ }^{44}$ L. Huang and J. Kieffer, Phys. Rev. B 69, 224203 (2004). 